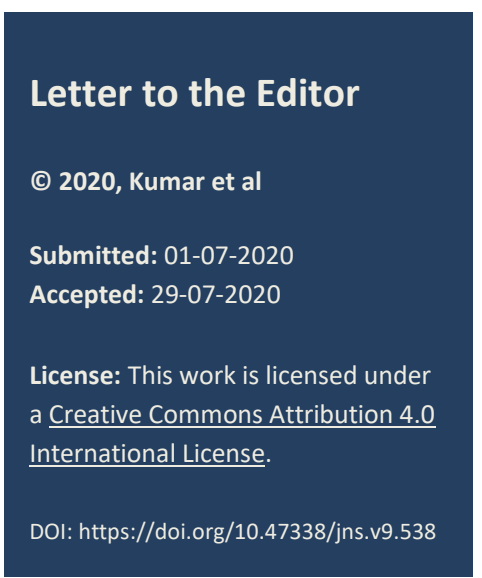

\section{Gastric perforation in a neonate with Hirschsprung disease}

Parveen Kumar, ${ }^{1}$ Yogesh Kumar Sarin, ${ }^{2}$ Shramana Mandal, ${ }^{3}$

1. Department of Pediatric Surgery, Chacha Nehru Bal Chikitsalya, New Delhi, India

2. Department of Pediatric Surgery, Lady Harding Medical College, New Delhi, India

3. Department of Pathology, Maulana Azad Medical College, New-Delhi, India

Correspondence*: Dr. Yogesh Kumar Sarin, Director Professor and Head Department of Paediatric Surgery Lady Harding Medical College, New Delhi, India. E-mail: yksarin@gmail.com

\section{Dear Editor}

Hirschsprung disease may present as neonatal bowel perforation. The site of perforation may vary depending on the segment of the bowel involved. Here we present a neonate that initially presented with a gastric perforation that was repaired. He later developed colonic perforation; colonic biopsies taken at second surgery proved the presence of Hirschsprung's disease.

A 2-day-old male term neonate born of uncomplicated pregnancy and normal vaginal delivery at a private hospital was referred to us with progressively increasing abdominal distension in the last 16 hours of life. He was breastfed once and had passed a small amount of meconium once at 10 hours of life. At presentation, he was dehydrated, tachypneic (RR $43 / \mathrm{min}$ ), tachycardia (HR 146/min), and had delayed capillary refill time (4 sec). Babygram showed a large amount of air under the diaphragm (Fig 1).

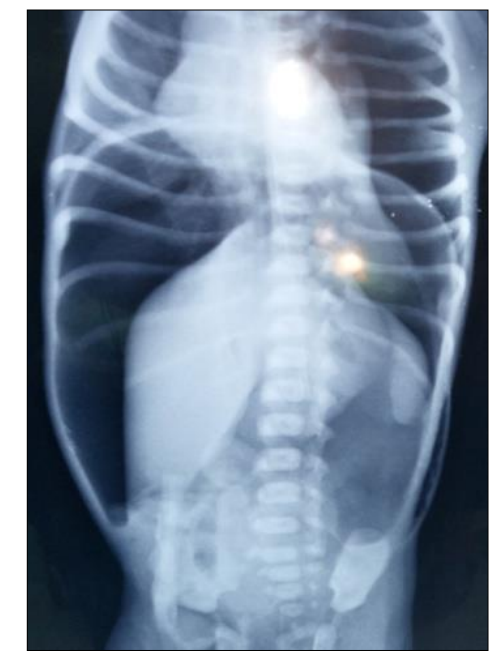

Figure 1: X-ray abdomen showing huge pneumoperitoneum.

Laboratory investigations were as: $\mathrm{Hb} 12.7 \mathrm{~g} \%$, total leukocyte counts of $18000 / \mathrm{cmm}, \mathrm{C}$ reactive proteins
$80 \mathrm{mg} / \mathrm{L}$, platelets $2.3 \mathrm{lacs} / \mathrm{cmm}$, urea $34 \mathrm{mg} / \mathrm{dl}$, creatinine $0.9 \mathrm{mg} / \mathrm{dl}, \mathrm{Na} 131 \mathrm{meq} / 1$ and $\mathrm{K} 2.3 \mathrm{meq} / 1$. Blood culture sent at admission was later found to be sterile. After initial resuscitation, he underwent exploratory laparotomy that revealed a large gastric perforation near the posterolateral wall of greater curvature (Fig 2). The rest of the bowel was healthy. The perforation site was primarily repaired.

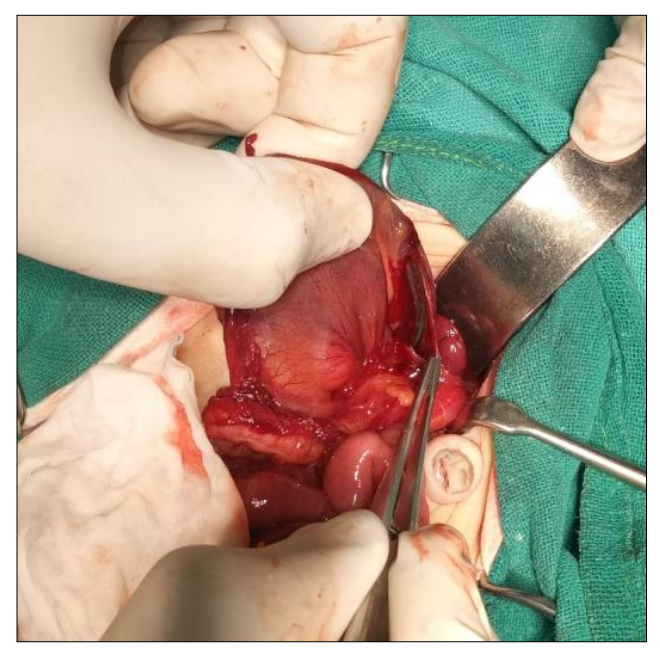

Figure 2: Operative picture showing gastric perforation.

On postoperative day 4 , he again started having abdominal distension. The septic profile revealed raised counts TLC $21000 / \mathrm{cmm}$, CRP $140 \mathrm{mg} / \mathrm{L}$, and falling platelets 1.2 lacs $/ \mathrm{cmm}$. Venous blood gas study revealed metabolic acidosis. On re-exploration, the gastric repair was found intact. Instead, a perforation in the left transverse colon was seen; no other abnormality in the small or large bowel could be appreciated. A divided stoma was created at the perforation site and multiple colonic biopsies were taken distal to the perforation. The baby succumbed after a few hours of re-exploration. Histopathology revealed aganglionosis in sigmoid colon and colo-rectal junction; ganglion cells were present at the site of perforation. 
Neonatal gastric perforation is a rare presentation. The cause may be traumatic, ischemic, or spontaneous. Iatrogenic nasogastric tube placement, especially in the setting of positive pressure ventilation may cause gastric perforations. The role of several gaps in circular muscle layers of neonates, especially in pretermers, has been postulated. 1 Ischemic gastric perforations have been described in necrotizing enterocolitis.2,3 An extensive literature search did not reveal any case of gastric perforation due to underlying Hirschsprung's disease. We hypothesize that gastric perforation was caused by early necrotizing enterocolitis or by iatrogenic injury due to the insertion of a nasogastric tube. The rapid deterioration could have

\section{REFERENCES}

1. Behrman RE, Kliegman RM, Jenson HB. In: Stoll BJ, Kliegman RM, editors. Digestive system disorders. Nelson textbook of pediatrics. 17 th ed. USA: Sanders; 2004:590-1.

2. Parasuraman D, Roy R, Mitchell S. Iatrogenic Gastric perforation in a preterm infant: clinical presentation been due to fungal sepsis as repeat culture sent at second surgery later grew Candida, which was inflicting our surgical neonatal ICU during that period.

Acknowledgments: Nil

Conflict of Interest: YKS is part of editorial team.

Source of Support: Nil

Consent to Publication: Author(s) declared taking informed written consent for the publication of clinical photographs/material (if any used), from the legal guardian of the patient with an understanding that every effort will be made to conceal the identity of the patient, however it cannot be guaranteed.

Author Contributions: Author(s) declared to fulfill authorship criteria as devised by ICMJE and approved the final version.

and radiological indicators. Internet $\mathrm{J}$ Pediatr Neonatol. 2006; 7:1.

3. Leone RJ, Krasna IH. Spontaneous neonatal gastric perforation: is it really spontaneous? J Pediatr Surg. 2000; 35:1066-9. 